\title{
Stellar population in bulge of spiral galaxies
}

\author{
L. Morelli ${ }^{1}$, E. Pompei ${ }^{2}$, A. Pizzella ${ }^{3}$, L. Coccato ${ }^{4}$, E.M.Corsini ${ }^{3}$, \\ J. Mendez ${ }^{3}$, R. Saglia ${ }^{5}$, M. Sarzi ${ }^{6}$ and F. Bertola ${ }^{3}$ \\ ${ }^{1}$ Pontificia Universidad Catolica de Chile \\ ${ }^{2} \mathrm{ESO} /$ Chile \\ ${ }^{3}$ Dip. Astronomia, Universita' di Padova \\ ${ }^{4}$ Kaptein Astronomical Institute \\ ${ }^{5}$ Max-Planck Institut für extraterrestrische Physik \\ ${ }^{6}$ University of Hertfordshire
}

\begin{abstract}
We present extensive photometric and spectroscopic study to give a new insight in the bulge stellar population. Super-solar $\alpha / \mathrm{Fe}$ and its constant value along the radial profile, in most of the galaxies, suggest that the star formation in these objects has been fast and occurred at the same time in the whole bulge.
\end{abstract}

\section{Introduction}

Bulge of spirals galaxies, due to their privileged position in the Galaxy structure, are very promising to reveal the mechanisms of formation and evolution of galaxies. Dynamical and kinematical studies have revealed their complexity suggesting they could have completely different nature trough the Hubble sequence. Chemical evolution studies on bulges of spirals are few and so far mainly based on field galaxies: they show that bulges hosted in early type spiral galaxies ( $\mathrm{Sa}$ to $\mathrm{Sb}$ ) behave similarly to field elliptical galaxies, while bulges hosted in later type spiral galaxies (Sc onward) host a younger stellar population. In this work we attack questions like the following:

- How do the bulges form?

Is the dissipative collapse, secular evolution, bar instability, merging events responsible for the bulge formation?

- How do the galaxies form?

Is the galaxy disk giving rise to the bulge or is the bulge a small elliptical acquiring a disk in the merging framework of galaxy formation?

Any good theory explaining the evolution of the universe must to be able to reproduce galaxies in agreement with the constrain given by the stellar population like age metallicity and $\alpha / \mathrm{Fe}$. The lack of observational clear constrain prevent so far a conclusive answer for the above questions. Here we derived metal abundances, age and $\alpha / F e$ of the stellar population for a large sample of galaxies, with a morphological type ranging from S0 to Sc, giving new fundamental clues to the challenge topic of the galaxy formation and evolution.

\section{Sample and observations}

The sample is composed by bright and nearby disk galaxies. All of them have been identified as member of the Fornax or Pegasus clusters and chosen to be unbarred with a low-to-intermediate inclination. We obtained long slit spectroscopy at the ESO 3.6 telescope using EFOSC2 spectrograph in the range between $4700 \AA$ and $6700 \AA$, with a instrumental $\mathrm{FWHM}=6.5 \AA$. For each galaxies we measured the kinematics for the stellar component and the radial profile along the major axis of the Lick indexes. 


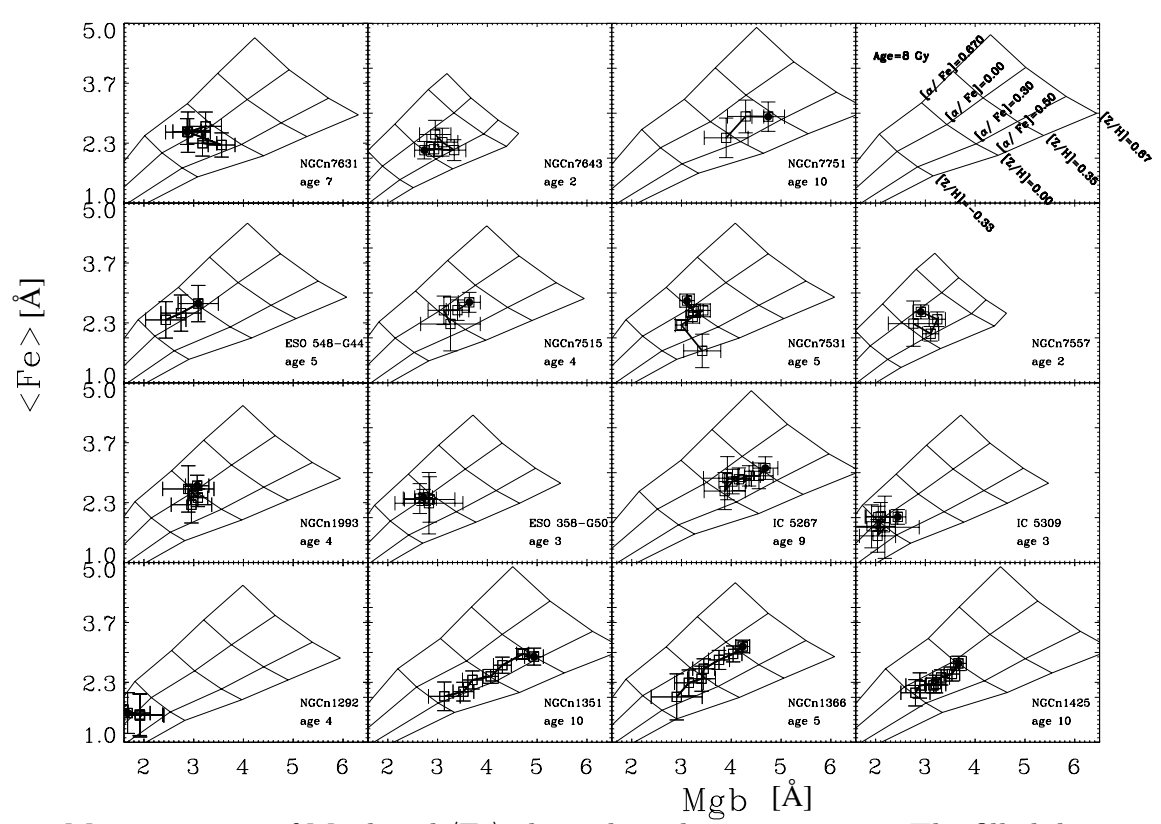

Figure 1. Measurements of $\mathrm{Mg} b$ and $\langle\mathrm{Fe}\rangle$ along the galaxy major axis. The filled dot represents the galaxy center. The model (Thomas et al. 2003) grid refer to the age of the galaxy. In the upper left panel are displayed the values for $\alpha / \mathrm{Fe}$ and metallicity in a grid corresponding to the fixed age of 12 Gyr.

\section{Results and conclusions}

In the results $\mathrm{Mg}_{2}$ and $\langle\mathrm{Fe}\rangle$, and therefore metallicity, show always their highest value in the center of the galaxy. Furthermore from the derived Lick indices and the velocity dispersion in the central regions of the bulges, we found a well defined relation of $\langle\mathrm{Fe}\rangle, \mathrm{Mg}$ $b$ and $\mathrm{H} \beta$ versus $\sigma$. This correlation is expected if the formation process of the spheroids occurred via dissipative collapse. Similar results have been found also for the Galactic bulge.

The constant and super-solar $(\alpha / \mathrm{Fe}=0.3)$ values of the $\alpha$-enhancement ratio along radius suggests that the formation of the bulk of the stars also in spiral bulges, as found in elliptical and S0 galaxies, occurred with the same short (less than 1 Gyr) timescale. Therefore the behavior of $\alpha /$ Fe radial profiles, disfavor strong inside-out or outside-in scenarios and are in conflict with the pure monolithic collapse, where a positive gradient in the $\alpha$-enhancement is expected (Martinelli et al. 1998). On the other hand the globally $\alpha /$ Fe enhanced stellar population (Thomas \& Kaufmann 1999) is not expected in the hierarchical merging scenario where the star formation is triggered by the merger event (Barnes 1992; Bender \& Surma 1992) and could give higher central values of $\alpha /$ Fe which decrease outwards (Thomas,1999).

\section{References}

Barnes, J. E. 1992 ApJ 393, 484

Bender, R. \& Surma, P. 1992 A $\&$ A 258, 250

Martinelli, A., Matteucci, F., \& Colafrancesco, S. 1998 MNRAS 298, 42

Thomas, D. 1999 MNRAS 306, 655

Thomas, D. \& Kauffmann, G. 1999 ASP-CS

Thomas, D., Maraston, C., \& Bender, R. 2003 MNRAS 339, 897 